\title{
Capturing the True Value of Assistive Technologies to Consumers in Routine Outcome Measurement
}

\author{
Desleigh de Jonge ${ }^{1,2, *}$ and Wendy Stevens ${ }^{1}$ \\ 1 LifeTec Australia, Brisbane 4051, Australia; wendystevens@lifetec.org.au \\ 2 School of Health and Rehabiliation Sciences, The University of Queensland, Brisbane 4072, Australia \\ * Correspondence: desleighdejonge@lifetec.org.au; Tel.: +61-7-3552-9000
}

Academic Editor: Jeffrey W. Jutai

Received: 25 May 2016; Accepted: 1 October 2016; Published: 14 October 2016

\begin{abstract}
Background: Recent reforms in Australia, providing people with disability and older people with choice and control over allocated funding, have altered consumer expectations and transformed the landscape of assistive technology (AT) service provision. The purpose of this study is to report on the routine AT outcomes of people who accessed an AT consultation service and examine how well these capture the impact of AT on their lives; (2) Methods: This study, which uses mixed methods for concurrent triangulation of the data, reports on the outcomes for 127 people who acquired a range of assistive technology in 2015 and examines the adequacy of an existing service outcome framework in capturing the true value of these technologies to AT users. Outcome data was routinely collected by a community service $2-4$ months following an AT consultation. A telephone or face-to-face interview gathered demographic information as well as AT outcomes, using two standardized tools, the Individualized Prioritised Problem Assessment (IPPA) and the EATS 6D. Qualitative comments relating to the impact of the AT on the person's life were also documented; (3) Results: The acquired AT generally met or exceeded expectations of the person using the AT and the attending health professional. Overall, people experienced decreased difficulty and increased feelings of autonomy, with most of the reported improvements identified in mobility and usual activities; (4) Conclusion: Routine outcome data provide some evidence of the value of AT in addressing concerns as identified by clients. Qualitative data, which captured the impact of AT on people's lives, suggest that the empowering and transformative aspects of AT are not currently being captured by existing measures.
\end{abstract}

Keywords: routine outcome measurement; assistive technology; service delivery

\section{Introduction}

People have traditionally accessed assistive technologies (AT) and related services in Australia via a patchwork of services and equipment schemes funded across three levels of government, namely Commonwealth, State and local governments as well as via various community organizations [1]. There are over one hundred separate AT funding schemes [2], which fund a limited range of devices either partially or fully, or alternatively provide devices on permanent loan, depending on the unique policies of each scheme [3]. Each of these schemes have specific eligibility criteria which vary widely across jurisdictions and service sectors [4]. Consequently, people with disabilities have had to rely heavily on professionals to navigate these schemes and provide adequate clinical justification in order to acquire assistive technologies.

To date Assistive Technology Services (ATS) have received block funding to provide services. Under this funding arrangement, services and the professionals who work in them have determined the nature of services provided and the focus of outcomes. 
ATS are currently preparing for radical changes in Australia in response to disability and aged care reforms that provide people with disability and older people with choice and control in identifying assistive technology (AT) solutions and use of funding. The National Disability Insurance Scheme (NDIS) Act (2013) "enables people with a disability to exercise choice and control in the pursuit of their goals in the planning and delivery of supports" [5] (p. 4). These changes shift the focus from a professional centric to a consumer directed process, requiring the benefits of AT to be described in terms that people understand and relate to so as to inform decision making and demonstrate effectiveness. Rather than professionals defining AT need in terms of lost function, people will articulate their goals and seek assistance from professionals to identify the AT that best achieves these goals.

Within this context, AT is recognized as a powerful enabler, empowering people to engage fully in society. This potential has been recognized in a recent definition where AT includes "technologies, equipment, devices, apparatus, services, systems, processes and environmental modifications used by older people or people with a disability to overcome the social, infrastructural and other barriers to independence, full participation in society and carrying out activities safely and easily" [6] (p. 196).

Traditionally, policy makers, funders and service providers have assumed the benefits of AT to be self-evident [7]. This has resulted in little attention being given by services to evaluating the effectiveness of these technologies [4,8,9]. More recently, the importance of measuring AT outcomes has been well documented [7], defining AT outcomes in terms of the changes that result in people's lives and their environments [7,10]. A number of frameworks and models have been developed to understand AT outcomes and describe the impact of AT on activities and participation as well as their economic benefits $[10,11]$. However, consumer research continues to identify hitherto uncaptured outcomes [10]. Consumers have reported AT as contributing to their independence [10], and participation in activities of daily living [11], work and school [10] as well as social [12] and leisure activities [13] subjective well-being [10], self-efficacy [14] and mobility-related community participation [15]. AT has also been found to decrease demands on caregivers [16].

Research on AT outcomes tends to be limited, small scale studies with small participant numbers. The range of AT under investigation and the intended purpose of the AT intervention also varies widely. These studies suggest that AT has many potential benefits to consumers, however there are numerous challenges to capturing AT outcomes [7,17] including the variability amongst AT users and their individual goals [8] and the limited availability and clinical utility of tools to measure AT outcomes [18].

AT outcomes are critical to decision-making and quality assurance in service delivery $[4,19]$. Numerous measures have been developed over the last 20 years to address different dimensions of AT outcomes [20]. However, in the absence of mandates to collect outcome data [7], the documentation of AT outcomes in service delivery is still in the early stages of development with AT professionals lacking procedures to routinely evaluate AT outcomes in practice [20-22]. Adopting a structured and user-centered approach to documenting AT outcomes in everyday practice has been proposed as a useful strategy for understanding the outcomes of AT interventions [20,23]. However, to date there have been few studies examining the effectiveness and suitability of existing outcomes measures for routinely measuring AT outcomes [20]. More importantly, limited attention has been given to whether these tools capture outcomes valued by consumers.

This study reports on AT outcomes routinely collected from people who acquired AT following a consultation with a dedicated AT service. It examines whether this routine data traditionally collected to report on outcomes to funding bodies adequately captures the impact of AT as experienced by the users.

\section{Materials and Methods}

This study uses mixed methods to report on routine AT outcomes for AT users following consultation with a dedicated AT advisory service. Descriptive analysis was undertaken on quantitative data using Excel TM. Content analysis was undertaken on qualitative data. Comments were read 
independently by each author and the main theme/s of each noted. The list of notes were reviewed by both authors and categorized. The statements were then reviewed again using categories and subcategories to ensure all statements were adequately captured by the categories. All data is used in accordance with the Australian Privacy Principles and the organization's privacy policy, which requires clients to provide informed consent on the use of data collected by the service. Participants provided consent for aggregated data related to routine services to be used for reporting and research purposes. The two authors, who work in senior positions at LifeTec, analyzed the service data. Neither author provided direct clinical services to these clients.

\subsection{Study Participants and Context}

Outcome data, routinely collected from clients who accessed AT consultation services from LifeTec over a twelve month period was examined for this study. Clients were people with a range of disabilities who were seeking assistance from a health professional to identify suitable AT. LifeTec is a publically funded AT information and advisory service that provides expert information, advice and education on assistive technologies and home modification and design. People generally self-refer and access the service to obtain appropriate documentation to secure subsidy funding for the identified AT from an appropriate government equipment scheme. The service employs health professionals (occupational therapists, physiotherapists and speech pathologist) who work with people to identify the best AT to address specific life goals. The health professionals use a range of standardized and non-standardized tools and clinical processes to determine the most suitable AT solution that best fits the client's person, occupation and environmental factors. In the initial interview, two standardized tools are also used with every client, namely the Individualized Prioritised Problem Assessment (IPPA) [24] and the EATS 6D (EATS 6 dimensions) [25] to identify issues; develop AT related goals and obtain measures of difficulty and autonomy. Where individuals are unable to provide responses to the attending health professional, information is collected from the family member or carer involved in the selection and application of the AT.

Approximately five hundred people accessed AT consultations in 2015. Everyone who accessed the service was routinely provided with a follow-up telephone or face-to-face interview two-four months following the AT Consultation by the attending health professional. This study examines data from a convenience sample of one hundred and twenty seven clients who had been using the AT for at least a few weeks and agreed to participate in a follow-up interview and complete the outcome measures.

\subsection{Outcome Measures}

During the follow-up interview, the health professional collected demographic information including each person's gender, living situation, age, health condition and existing AT. Information was also collected on whether the person had acquired the recommended AT and if not, the reason for not acquiring it. For those who had acquired the AT, the type of AT was recorded. AT outcomes were collected in the follow-up interview using two standardized tools, the Individualized Prioritised Problem Assessment (IPPA) and the EATS 6D. Both of these tools are user-centered and were specifically designed to collect data directly from the real-life experiences of the AT user. At the end of the interview people were asked to describe the impact that the AT had on their lives. The statements were transcribed by the attending health professional at the time of the interview. All questions were directed to the individual who had received the service to meet an AT need by the attending health professional. For individuals who were unable to communicate for themselves, responses were provided by the family member or carer involved in the selection and use of the assistive technology.

The Individualized Prioritized Problem Assessment (IPPA) [24] was specifically designed to assess the impact of assistive devices as existing instruments in the field of Health Technology Assessment did not adequately assess the effectiveness of assistive technology [26]. This tool was chosen as it provides clinicians with a standardized way to understand and document the presenting problems 
and difficulties being experienced as well as whether the acquired AT met their expectations. This tool provides a list of daily activities, namely self-care, mobility, transportation, housework, safety, leisure, communication, work and social interaction as a foundation for discussing potential problems with clients. It enables issues to be prioritized and the scores at the initial interview to then be compared with scores following acquisition of the device. In the initial and follow-up interview the IPPA was used by the attending health professional to identify problems and rate the importance and degree of difficulty the person experiences in carrying out the activity on a seven-point scale, with 1 being of no importance at all/not at all difficult and 7 being most important/too difficult to perform activity. In this study, comments provided by the person using the AT (or their family member/carer) about difficulty were also transcribed by the health professional at the time of the interview.

The IPPA also asks the person to rate how the AT has addressed each identified problem on a five-point scale, with -2 being much less than expected and +2 being much more than expected. In this study, comments provided by the person regarding the extent to which the AT met their expectations were also transcribed by the health professional at the time of the interview. The health professionals also recorded the extent to which the AT had met their clinical expectations using this scale and where relevant, provided notes on how expectations might be addressed. This tool has been found to be a useful individualized measure for evaluating AT device and service delivery outcomes [27].

The EATS 6D (EATS 6 dimensions) [25] describes changes in autonomy in daily life following the acquisition of AT. It was also used in the initial and follow-up interview. The tool asks people to indicate the extent to which they feel autonomous by pointing to a visual analogue scale (0-100 in increments of 10) with 0 being the worst imaginable state and 100 being the best imaginable state after being asked "Today I feel ... ". At all initial interviews, individuals were shown the visual analogue scale. During the follow-up interviews conducted over the phone, individuals were reminded of the scale and asked to indicate the extent to which they feel autonomous on a scale of 0 to 100 with 0 being the worst imaginable state and 100 being the best imaginable state. The EATS-6D also examines individual perception of autonomy in six dimensions of daily life namely mobility, self-care, usual activities, pain/discomfort, anxiety/depression and relationships. In addition, each person was asked to describe how the AT had impacted on his/her life.

Construct validity for both the IPPA and EATS 6-D was established with score changes being consistent with the SIP68 and EuroQol. Test-retest reliability for the IPPA was found to be best when the same assessor was used. Test-retest and inter-assessor reliability of the EATS 2-D scale was found to be medium to fair [28].

\subsection{Data Management}

Due to the limited reliability of the IPPA and EATS-6D, pre and post scores for were compared and recorded as to whether there had been no change in score or whether there had been an increase or decrease in scores between the initial and follow-up interview.

\section{Results}

Approximately $80 \%$ of the follow-up interviews were conducted over the phone, with the remaining $20 \%$ being undertaken face-to-face. Outcome data was recorded on one hundred and twenty seven people, approximately forty percent of the people who accessed an AT consultation. Just over half of the study participants were female (56.15\%). Most lived with family or others (61.83\%), whilst some lived alone $(23.66 \%)$ or in formal care $(6.11 \%)$. Information was not available on the living situation of the remaining people $(8.40 \%)$. The majority of people accessing the consultation services were over 50 years old. Detailed demographic information is available in Table 1. 
Table 1. Sample Characteristics.

\begin{tabular}{|c|c|c|}
\hline Age Range $(n=106)$ & $f$ & $\%$ \\
\hline $65+$ & 30 & 28.30 \\
\hline $55-64$ & 27 & 25.47 \\
\hline $45-54$ & 18 & 16.98 \\
\hline $35-44$ & 11 & 10.38 \\
\hline $25-34$ & 5 & 4.72 \\
\hline $15-24$ & 8 & 7.55 \\
\hline$<15$ & 7 & 6.60 \\
\hline \multicolumn{3}{|l|}{ Condition $(n=118)$} \\
\hline Neurological & 20 & 16.95 \\
\hline Arthritis & 15 & 12.71 \\
\hline Multiple Sclerosis & 13 & 11.02 \\
\hline Physical & 9 & 7.63 \\
\hline Cerebral Palsy & 8 & 6.78 \\
\hline Frail Aged & 8 & 6.78 \\
\hline Intellectual & 7 & 5.93 \\
\hline Spinal Cord Injury & 6 & 5.08 \\
\hline Vision & 6 & 5.08 \\
\hline Muscular Dystrophy & 4 & 3.39 \\
\hline Parkinsons Disease & 4 & 3.39 \\
\hline Chronic Disease & 3 & 2.54 \\
\hline CVA & 3 & 2.54 \\
\hline $\mathrm{ABI}$ & 3 & 2.54 \\
\hline Motor Neurone Disease & 2 & 1.69 \\
\hline Spina Bifida & 2 & 1.69 \\
\hline Alzheimer & 1 & 0.85 \\
\hline Autism & 1 & 0.85 \\
\hline Cancer & 1 & 0.85 \\
\hline Hearing & 1 & 0.85 \\
\hline Back or Neck Pain & 1 & 0.85 \\
\hline
\end{tabular}

Most people were already using one $(43.85 \%)$ or more $(42.30 \%)$ device types at the time of the initial consultation. Only a small number $(14.00 \%)$ were not currently using a device. A broad range of devices were in use prior to the AT consultation. Mobility devices were the most common device type in use followed by self-care and hygiene equipment; seating and positioning devices; hoisting/transfer devices, and pressure care cushions and mattresses. Home modification, vehicle and computer access devices, personal alarms, and vision related AT were also being used, as were a small number of devices related to community access, communication, telecommunication, recreation and hearing.

\subsection{AT Acquisition and Follow-Up}

At follow-up, ninety-four people (74.02\%) had acquired the recommended AT. Five people (3.93\%) had acquired AT but not the item that had been recommended. Nineteen (14.96\%) were still planning to acquire the AT but had not yet done so, whilst two were continuing with their existing AT $(1.57 \%)$ and seven $(5.50 \%)$ were not planning to proceed with the AT recommendation at this point. One commented that they were not proceeding because the client had passed away in the interim.

\subsection{Why AT Was Not Acquired}

Of the twenty-eight people who had not acquired AT at follow-up, nineteen $(67.78 \%)$ were still planning to, seven $(25.00 \%)$ were not planning to proceed and two $(7.14 \%)$ continued to use their existing AT. For the nineteen who were still planning to acquire the AT, the reasons for delay included not being able to afford the AT (21.05\%), waiting for funding approval $(15.79 \%)$, waiting to trial the AT $(15.79 \%)$ or waiting to see another health professional. Three of the people who were not planning to proceed indicated that this was because they were unable to afford the AT, whilst the health condition 
of the remaining four had deteriorated (including one person who had since passed away). The two who continued to use their existing AT did so because they were yet to find a suitable solution.

Other reasons provided for not acquiring the AT included: being busy, confused about the next step, or experiencing difficulty in changing to a new way of undertaking the task.

\subsection{Type of AT Acquired}

Eighty-five people had acquired AT at the time of the follow-up call with $77.64 \%$ acquiring one device, $18.82 \%$ two devices and three people acquiring three devices. Mobility AT was the most frequently acquired AT, being $45.37 \%$ of all devices acquired. A detailed list of AT types acquired is described in Table 2.

Table 2. Type of AT acquired.

\begin{tabular}{lcc}
\hline \multicolumn{1}{c}{ Type of AT Acquired $(\boldsymbol{n = 1 0 8 )}$} & $f$ & $\mathbf{\%}$ \\
\hline Mobility all & 49 & 45.37 \\
Mobility-Scooter & 20 & 18.52 \\
Mobility-Manual Wheelchair & 15 & 13.89 \\
Mobility-Walker & 7 & 6.48 \\
Mobility-Power Wheelchair & 4 & 3.70 \\
Mobility-Other & 3 & 2.78 \\
Vehicle access/transfers & 15 & 13.89 \\
Recreation & 8 & 7.41 \\
Computer Access/Switch/Mainstream Technologies & 7 & 6.48 \\
Community Access & 6 & 5.56 \\
Home Modifications & 5 & 4.63 \\
Seating, standing \& positioning & 4 & 3.70 \\
Magnifiers/Vision AT & 4 & 3.70 \\
Self Care & 4 & 3.70 \\
Hoisting/Transfers & 3 & 2.78 \\
Smart Phones/Telecommunications & 2 & 1.85 \\
Communication & 1 & 0.93 \\
\hline
\end{tabular}

\subsection{Identified Problems (IPPA)}

Most people sought an AT consultation to address one problem (77.78\%) however, $18.89 \%$ identified two, 2.22\% identified three and $1.11 \%$ identified four problems to address using AT.

The acquired AT generally met $(50.00 \%)$ or exceeded expectations $(43.63 \%)$ for the person using the AT. From the health professional's perspective, the acquired AT also met $(64.21 \%)$ or exceeded expectations $(30.53 \%)$. For the $6.36 \%$ people whose expectations were not met, $64.00 \%$ had also acquired other AT at that time which had met (two people) or exceeded (one person) expectations. One person's comment revealed that the he had difficulty using the AT with other equipment within the workplace. In the remaining four cases, where the individual was only recommended one AT, comments indicated that the person was waiting on modifications to be made to the home before the AT could be used, experiencing pain when using the device or that expectations of the device had grown once he started using it.

Suggestions were provided by the health professionals on how consumer expectations might be better met. These included more supportive suppliers, decreasing the weight (2) and cost of the equipment, receiving more feedback from the family during the trial process, greater co-ordination across service providers working on different issues and ensuring the individual understood the importance of including all recommended elements/accessories to achieving the goal.

For $79.70 \%$ of the people who had acquired their AT, the degree of difficulty score at follow-up was lower than at the initial assessment whilst in $12.00 \%$ the AT did not make any difference to the difficulty the individual experienced with this problem in everyday life. For some (12.70\%), there was an increase in difficulty following the introduction of AT. 


\subsection{Autonomy (EATS-6D)}

Most people (52.70\%) experienced an increase in feelings of overall autonomy whilst some felt the same $(31.08 \%)$ or less autonomy $(16.22 \%)$. No change was recorded for the majority of people across all six domains however over half (56.65\%) experienced an improvement in one or more with most of the reported improvements in mobility and usual activities.

\subsection{Changes Resulting from AT}

The participants reported AT as having a range of impacts as visualized in Figure 1. The key themes are represented by the increased size and intensity of color of the text. The frequency of each theme is provided in parentheses.

\section{community engagement confidence easier freedom independence less fatigue less pain aliberating safer social engagement}

Figure 1. Tag cloud of content analysis of comments.

\subsubsection{Community and Social Engagement (23)}

The predominant theme regarding the changes AT had made to their lives was improved community engagement.

[The mobility scooter is] great, I can now take my son to the beach, bike riding and out fishing.

I am also able to take my son to school and access the shopping centre ... It's all going well.

People were able to visit places they had previously been unable to go to, as evidenced by one person's comment.

She is able to access the bigger shopping centres where she hasn't been for a long time.

[family member/carer of a person using a manual wheelchair]

The extent to which the AT allowed them to get out and about exceeded one person's expectations.

It has surprised me how much more I am able to get out now. [using a mobility scooter]

This increased community mobility also afforded greater social engagement with people being able to visit family and friends and go out with them to a range of places and events.

Given more access to doing a lot more things with friends/family in the community. [using a manual wheelchair]

This allowed them to develop important relationships.

I can go out with my grandson-just the two of us. [using a mobility scooter]

Increased community and social engagement also had a dramatic impact on individuals' health and wellbeing, allowing them to engage in healthful activities. More importantly, it also enabled them to do things many able-bodied people take for granted. 
The funding that has been provided [for my manual wheelchair] has changed my/our lives dramatically. I am able to get outdoors sooo much easier ... and I am keen to. I feel like I can do things and be like other people and that makes such a difference. There is so much that I can't do but also so much I can do. I love going out and spending time outdoors and I love swimming. You have all made such a difference in my life and I wouldn't have been able to purchase myself due to financial constraints.

\subsubsection{Liberating ... Freedom (16)}

AT freed people to engage in everyday activities without having to make complex and intrusive arrangements.

I don't even think about it anymore-I just go. Whereas before, I had to think about my back pain and the practicality of everything. [vehicle modification]

This freedom also resulted in feelings of elation as people felt like they could be in the mainstream of life.

When I'm out on the trike, I'm treated as a health nut, which is totally different to being on a scooter, where you're invisible ... I feel like I'm just like everyone else ... Back in mainstream life ... freedom! [using a specialized tricycle]

One person described this feeling and the resulting impact on his/her mood and sense of wellbeing in the following comment: Liberating, very liberating ... Makes you more positive. [using a mobility scooter] Others also communicated the pervasive impact of their AT, saying:

Couldn't live without it. [using a transfer device]

She loves it. [family member/carer of a person using a manual wheelchair]

It's changed my life a lot-it's given me some life back... It's been fantastic. [using a scooter]

\subsubsection{Easier (13)}

Many reported that their AT had made things (tasks/life) easier for them with numerous comments beginning with: It has made it much easier...

Being able to undertake activities more easily is particularly important for carers, ensuring the viability of them providing informal care in the long term.

So much easier to get (my son) in and out of the car ... He has put on about $5 \mathrm{~kg}$ so it has saved our backs having the vans. [family member/carer of a person using a vehicle hoist]

\subsubsection{Independence (13)}

Being able to undertake activities without the assistance of others was also a highly valued outcome of AT.

\section{It has changed it (my life) immensely; I can do so much more independently. [using a magnifier]}

For some, the AT enabled them to undertake new activities as they were no longer reliant on others being available to assist them.

Doing things he hasn't had the chance to do before and providing more independence.

[family member/carer of a person using a power wheelchair]

Importantly, independence also affords people privacy as they are not dependent on others to assist them with personal activities.

I am now able to read my printed mail completely independently and maintain my privacy.

[using Vision AT] 


\subsubsection{Safer (9)}

A number of people commented that the AT made it safer for them to undertake various activities as reflected in the following comments

I feel much safer when getting into the shower. [Hygiene AT]

[The walker] has made me safer mobilising around the home.

I can access the community more safely and more comfortably using the new stroller.

\subsubsection{Confidence (5)}

AT also allowed people to feel more confident.

(I am) happy to go places ... Increased confidence. [using a manual wheelchair]

This improved confidence can then increase an individual's motivation to do things.

Increasing confidence when walking-wants to be able to do it independently and motivated to keep trying! [family member/carer of a person using a walker]

This enabled them to be more active reducing the enduring health consequences of inactivity as evidenced in the following comments.

(The person) now feels more confident in moving around own home, rather than staying in one seat.

The Bike has made me more active. [family member/carer of a person using a specialized bike]

\subsubsection{Reduced Fatigue and Pain $(4+2)$}

For some the predominant impact of AT on their lives was the reduction in fatigue and pain.

I do not need to rest as much when working. [using specialized seating]

The new office chair has reduced fatigue when sitting and improved comfort. [using an office chair]

Much less pain and it's much easier to stand up. I use it every day. [using specialized seating]

\section{Discussion}

This study examined the outcomes for AT users from AT recommended by a dedicated AT service. It is evident that most people acquired the recommended AT, but given the limited uptake of follow-up interviews, it is not certain that this reflects the actions of all service users. The use of a client-centered tool such as the IPPA to identify issues looks to have been useful in addressing the specific concerns of the client. Previous research on abandonment has highlighted the importance of involving the client in the process [29]. The comprehensive overview of all available AT suited to the individual's requirements provided by a dedicated, publicly funded service is also likely to ensure each individual is fully aware of their options and had the opportunity to discuss the potential strengths and limitations of each with the health professional. Having a clear understanding of needs prior to investigating technology options and access to comprehensive information on options has been identified previously by consumers as being essential to the process of identifying the best solution [8].

AT acquisition is not a predictable process. Delays make it difficult for health professionals to co-ordinate follow-up interviews and possibly impact client willingness to engage in these interviews. Acquisition was found to be hampered by a number of factors. Firstly, long delays in acquiring some AT types resulted from the extended time required for funding approval or because people had to raise the funds themselves. Constraints and delays in funding have long been identified as an enduring problem in AT service delivery [10]. Secondly, health complications and life stresses also prolonged the acquisition process and changed priorities. The changing needs of AT users have previously been noted to affect the AT process $[30,31]$. Variability in health and function can result in complexities for systems that have rigid definitions of disability, eligibility criteria, processes and funding guidelines. 
Challenges experienced by people in accessing and navigating supply of AT have not been examined extensively in the literature to date. It is important that these delays do not persist under the NDIS.

AT met the expectations of AT users and health professionals similarly, although more people using the AT reported expectations being exceeded than for the health professionals. It is possible that health professionals, experienced in recommending AT, have a well-developed understanding of what the AT can achieve and therefore have well-defined expectations. The person using the AT might not have experience that allows them to anticipate the potential benefits of the new device. The qualitative responses suggest that the AT did more than address a difficulty, providing a range of unexpected benefits.

As might be expected, appropriately selected AT reduced the difficulty people experienced in carrying out activities of interest. However changing or introducing new AT can change in the way the activity is undertaken or require the person to learn new skills. In some cases, the new AT may not work well with other devices or fit well within the environment. Whilst some of these complexities can be anticipated by an experienced user or health professional, there is sometimes a domino effect when new devices are introduced. This highlights the need for follow-up to provide required support and make the necessary adjustments to resolve issues. The need for follow-up continues to be promoted in the literature as being essential to quality AT service delivery [8,32-34], but the benefits of or barriers to undertaking follow-up have not been extensively researched.

Only half of the people using AT indicated an increase in feelings of autonomy. Autonomy is often affected by a range of factors, and the EATS did not specifically ask about the impact of AT on their feelings of autonomy, so people's scores might relate to generally feelings of powerlessness that are not specifically related to the AT. Responses to a general question of this nature may also vary from day to day, depending on recent experiences. People might also struggle to answer questions about their sense of autonomy, as autonomy may not be something people think about. Responses to the question on the impact of AT on their lives suggest that changes in autonomy are not being adequately reflected in EATS 6D.

Comments provided by people using AT reflects the transformative impact of AT on their lives. Even the brief descriptions of social and community engagement suggested the value people placed on engaging with loved ones and other community members; accessing community facilities, events and attractions; and the resulting health and well-being that resulted from the relationships and encounters experienced. The freedom and liberation afforded by AT, allowing people to engage in mainstream activities, released people from arduous arrangements and transformed their outlook. The independence and ease of performing tasks not only reduced reliance on others and demand on carers, it also increased the range of activities that people could engage in and the viability of carers providing ongoing support. AT was also identified as improving safety, confidence and reducing fatigue and pain, which then increased motivation to become more active. The results of this study echo findings of other qualitative inquiries that have highlighted the personal outcomes valued by AT users [10,14,31].

Traditional tools looking for functional gains or improvements in activities and participation risk masking the contribution AT can make to people's physical, emotional and social well-being. In consumer-directed service environments, the benefits of AT need to be described to users in ways that resonate with them [10,35]. It is imperative therefore that outcome methodologies reflect consumer priorities [6] if we are to capture the true value of AT to the people who rely on these powerfully enabling devices.

\subsection{Limitations of the Study}

Limited evidence on the psychometric properties of the outcome measures used in this study could have an impact on the validity of the quantitative data. Difficulties contacting people for follow-up and time constraints of the attending health professional meant that data was not available on all clients. Further, as the professional was aiming to interview the person after they had been using 
their AT for at least a few weeks, long delays in funding approval and AT delivery prevented many people from being interviewed. Although health professionals were strongly encouraged to obtain outcome data, it was not a mandatory requirement of the organization or the organizations' funding bodies. Clients were also able to decline to complete the outcome measures. This was often the case when the device was not considered complex or external funding was not required. People without an effective means of communication were not able to complete the initial assessment. Consequently, views and experiences of people who agreed to participate in the follow-up interview may not reflect those of all people accessing the service. With the attending therapist undertaking the follow-up interview, there is also likely to be a positive bias in the reported data. With most of the follow-up interviews being conducted over the phone, the reliability of the outcome data collected via the two standardized tools is likely to be affected.

\subsection{Future Research}

Further research is required to understand the impact of AT on people's lives, in particular their well-being, and how important it is for health professionals and consumers to consider this when selecting and implementing AT. Since the impact of AT devices in this study was assessed mostly for mobility devices, the impact of using other types of AT devices and the differences among the impact of different types of AT devices and for different groups of users require further exploration. Furthermore it is important that the clinical utility of AT outcome tools is examined carefully if they are to be used routinely in practice. Tools that can be used over the phone or over the internet are likely to improve convenience for clients and health professionals and increase response rate when gathering AT outcomes.

\section{Conclusions}

This study demonstrates the usefulness of integrating outcome measurement into AT service delivery and highlights the importance of the clinical utility of outcome measures in promoting routine data collection. It draws attention to the value of using client-centered tools in engaging people in the process of AT selection. The potential transformative impacts of AT for people who use them, evidenced by qualitative comments, highlights the need for outcome frameworks and tools to capture the true value of AT for consumers.

Acknowledgments: LifeTec receives public funding from state and federal government agencies to provide clinical assistance to people regarding their assistive technology. This study was undertaken to inform service delivery and development and assure quality of service provision. We are grateful to everyone who participated in the follow-up interviews. No grants were accessed to support this study.

Author Contributions: Desleigh de Jonge and Wendy Stevens analyzed the data and wrote the paper. Both authors have read and approved the final manuscript.

Conflicts of Interest: The authors declare no conflict of interest.

\section{References}

1. Layton, N.; Steel, E.; de Jonge, D. Choice and Control: Assistive Technology within Australia's New National Disability Insurance Scheme 2013. In Assistive Technology: From Research to Practice; Encarnação, P., Azevedo, L., Gelderblom, G.J., Newell, A., Mathiassen, N.-E., Eds.; IOS Press: Amsterdam, The Netherlands, 2013; Volume 33, pp. 266-272.

2. Summers, M. Ripe for reform: Aids and equipment policy. Health Issues 2011, 105, 32-34.

3. Pearson, J.; O'Brien, K.; Hill, S.; Moore, D. Research for the National Disability Agreement Aids and Equipment Reform: Final Report; FaHCSIA: Canberra, Australia, 2013.

4. Layton, N. Barriers and facilitators to community mobility for assistive technology users. Rehabil. Res. Pract. 2012. [CrossRef] [PubMed]

5. National Disability Insurance Scheme Act 2013; Australian Government: Canberra, Australia, 2013. 
6. Hersh, M.A.; Johnson, M.A. On modelling assistive technology systems-Part I: Modelling framework. Technol. Disabil. 2008, 20, 193-215.

7. Fuhrer, M.J.; Jutai, J.W.; Scherer, M.J.; DeRuyter, F. A framework for the conceptual modeling of assistive technology device outcomes. Disabil. Rehabil. 2003, 25, 1243-1251. [CrossRef] [PubMed]

8. De Jonge, D.; Scherer, M.; Rodger, S. Assistive Technology in the Workplace; Elsevier Mosby: St. Louis, MO, USA, 2007.

9. Rust, K.L.; Smith, R.O. Assistive technology in the measurement of rehabilitation and health outcomes. Am. J. Phys. Med. Rehabil. 2005, 84, 780-793. [CrossRef] [PubMed]

10. Lenker, J.A.; Harris, F.; Taugher, M.; Smith, R.O. Consumer perspectives on assistive technology outcomes. Disabil. Rehabil. Assist. Technol. 2013, 8, 373-380. [CrossRef] [PubMed]

11. Schraner, I.; de Jonge, D.; Layton, N.; Bringolf, J.; Molenda, A. Using the ICF in economic analyses of Assistive Technology systems: Methodological implications of a user standpoint. Disabil. Rehabil. 2008, 30, 916-926. [CrossRef] [PubMed]

12. Lee, S.H. Users' satisfaction with assistive devices in South Korea. J. Phys. Ther. Sci. 2014, 26, 509-512. [CrossRef] [PubMed]

13. Lancioni, G.E.; O’Reilly, M.F.; Singh, N.N.; Sigafoos, J.; Boccasini, A.; La Martire, M.L.; Perilli, V.; Spagnuolo, C. Technology to support positive occupational engagement and communication in persons with multiple disabilities. Int. J. Disabil. Hum. Dev. 2016, 15, 111-116. [CrossRef]

14. Folan, A.; Barclay, L.; Cooper, C.; Robinson, M. Exploring the experience of clients with tetraplegia utilizing assistive technology for computer access. Disabil. Rehabil. Assist. Technol. 2015, 10, 46-52. [CrossRef] [PubMed]

15. Sund, T.; Iwarsson, S.; Anttila, H.; Brandt, Å. Effectiveness of powered mobility devices in enabling community mobility-related participation: A prospective study among people with mobility restrictions. Am. J. Phys. Med. Rehabil. 2015, 7, 859-870. [CrossRef] [PubMed]

16. Mortenson, W.B.; Demers, L.; Fuhrer, M.J.; Jutai, J.W.; Lenker, J.; DeRuyter, F. Effects of an assistive technology intervention on older adults with disabilities and their informal caregivers: An exploratory randomized controlled trial. Am. J. Phys. Med. Rehabil. 2013, 92, 297-306. [CrossRef] [PubMed]

17. Lenker, J.A.; Scherer, M.J.; Fuhrer, M.J.; Jutai, J.W.; DeRuyter, F. Psychometric and administrative properties of measures used in assistive technology device outcomes research. Assist. Technol. 2005, 17, 7-22. [CrossRef] [PubMed]

18. Edyburn, D.L. Expanding the use of assistive technology while mindful of the need to understand efficacy. In Efficacy of Assistive Technology Interventions; Edyburn, D.L., Ed.; Emerald Group Publishing: Bingley, UK, 2015; Volume 1, pp. 1-12.

19. Douglas, H.; Swanson, C.; Gee, T.; Bellamy, N. Outcome measurement in Australian rehabilitation environments. J. Rehabil. Med. 2005, 37, 325-329. [CrossRef] [PubMed]

20. Desideri, L.; Bizzarri, M.; Bitelli, C.; Roentgen, U.; Gelderblom, G.J.; de Witte, L. Implementing a Routine Outcome Assessment Procedure to Evaluate the Quality of Assistive Technology Service Delivery for Children with Physical or Multiple Disabilities: Perceived Effectiveness, Social Cost, and User Satisfaction. Assist. Technol. 2015, 28, 30-40. [CrossRef] [PubMed]

21. Parette, H.P.; Peterson-Karlan, G.R.; Smith, S.; Gray, T.; Silver-Pacuilla, H. The state of assistive technology: Themes from an outcomes summit. Assist. Technol. Outcomes Benefits 2006, 3, 15-33.

22. Steel, E.J.; de Witte, L.P. Advances in European Assistive Technology service delivery and recommendations for further improvement. Technol. Disabil. 2011, 23, 131-138.

23. Phillips, B.; Zhao, H. Predictors of assistive technology abandonment. Assist. Technol. 1993, 5, 36-45. [CrossRef] [PubMed]

24. Wessels, R.; Persson, J.; Lorentsen, O.; Andrich, R.; Ferrario, M.; Oortwijn, W.; VanBeekum, T.; Brodin, H.; de Witte, L. IPPA: Individually Prioritised Problem Assessment. Technol. Disabil. 2002, 14, 141-145.

25. Andrich, R.; Ferrario, M.; Wessels, R.; de Witte, L.; Persson, J.; Oberg, B.; Lorentsen, Ø. Assessing Outcomes of Technology Products and Services: The Eats Instruments; EUSTAT Consortium: Milano, Italy, 1998.

26. Wessels, R.; Witte, L.D.; Andrich, R.; Ferrario, M.; Persson, J.; Oberg, B.; Oortwijn, W.; VanBeekum, T.; Lorentsen, Ø. IPPA, a user-centred approach to assess effectiveness of assistive technology provision. Technol. Disabil. 2000, 13, 105-115. 
27. Wessels, R.D.; De Witte, L.P.; Jedeloo, S.; van den Heuvel, W.J. A. Effectiveness of provision of outdoor mobility services and devices in the Netherlands. Clin. Rehabil. 2004, 18, 371-378. [CrossRef] [PubMed]

28. Persson, J.; Hellbom, G.; Oberg, B.; van Beekum, T.; Oortwijn, W.; Brodin, H.; Lorentsen, O.; Renzo Andrich, R.; Ferrario, M.; de Witte, L.; et al. Final Report: Efficiency of Assistive Technology and Services; 2000 EUSTAT Consortium: Milano, Italy, 2000.

29. Martin, J.K.; Martin, L.G.; Stumbo, N.J.; Morrill, J.H. The impact of consumer involvement on satisfaction with and use of assistive technology. Disabil. Rehabil. Assist. Technol. 2011, 6, 225-242. [CrossRef] [PubMed]

30. Demers, L.; Fuhrer, M.J.; Jutai, J.W.; Scherer, M.J.; Pervieux, I.; DeRuyter, F. Tracking mobility-related assistive technology in an outcomes study. Assist. Technol. 2008, 20, 73-85. [CrossRef] [PubMed]

31. Gramstad, A.; Storli, S.L.; Hamran, T. Exploring the meaning of a new assistive technology device for older individuals. Disabil. Rehabil. Assist. Technol. 2014, 9, 493-498. [CrossRef] [PubMed]

32. Bauer, S.; Elsaesser, L.J.; Scherer, M.; Sax, C.; Arthanat, S. Promoting a standard for assistive technology service delivery. Technol. Disabil. 2014, 26, 39-48.

33. Cook, A.M.; Polger, J.M. Assistive Technologies: Principles and Practice, 4th ed.; Elsevier Mosby: St Louis, MO, USA, 2015.

34. Federici, S.; Borsci, S. Providing assistive technology in Italy: The perceived delivery process quality as affecting abandonment. Disabil. Rehabil. Assist. Technol. 2016, 11, 22-31. [CrossRef] [PubMed]

35. Dijkers, M. Issues in the conceptualization and measurement of participation: An overview. Arch. Phys. Med. Rehabil. 2010, 91, S5-S16. [CrossRef] [PubMed]

(C) 2016 by the authors; licensee MDPI, Basel, Switzerland. This article is an open access article distributed under the terms and conditions of the Creative Commons Attribution (CC-BY) license (http:/ / creativecommons.org/licenses/by/4.0/). 